\title{
Hypoxia and Outcome Prediction in Early-Stage Coma (Project HOPE): an observational prospective cohort study
}

Alex Lopez-Rolon and Andreas Bender ${ }^{1,2}$ on behalf of the Project HOPE Investigator Group

\begin{abstract}
Background: The number of resuscitated cardiac arrest patients suffering from anoxic-ischemic encephalopathy is considerable. However, outcome prediction parameters such as somatosensory evoked potentials need revision because they are based on data predating the implementation of mild therapeutical hypothermia and because data from our own laboratory suggest that they may fail to predict prognosis accurately. The present research project "Hypoxia and Outcome Prediction in Early-Stage Coma" is an ongoing observational prospective cohort study that aims to improve outcome prediction in anoxic coma by limiting the effects of falsely pessimistic predictions at the intensive care unit.

Methods: Our outcome analysis is based on functional and behavioural definitions. This implies the analysis of the positive predictive value of prognostic markers yielding either positive or negative results. We also analyse the effect of covariates adjusted for age and sex such as sociodemographic variables, prognostic variables and treatment factors on functional and behavioural outcomes, with mixed effects regression models (i.e. fixed and random effects). We expect to enrol 172 patients based on the result of previous research. The null hypothesis is that there is a probability of $<10 \%$ that a positive outcome will be observed despite the presence of any of the predictors of a poor/negative outcome. We test the null hypothesis against a one-sided alternative using a Simon's two-stage design to determine whether it is warranted to recruit the full number of patients suggested by a power analysis. The second stage has a design with a Type I error rate of 0.05 and $80 \%$ power if the true response rate is $25 \%$.

Discussion: We aim to make a significant contribution to the revision and improvement of current outcome prediction methods in anoxic-ischemic encephalopathy patients. As a result, neurocritical care specialists worldwide will have considerably more accurate methods for prognosticating the outcome of anoxic-ischemic encephalopathy following cardiac arrest. This will facilitate the provision of treatment tailored to individual patients and the attainment of an optimal quality of life. It will also inform the decision to withdraw treatment with a level of accuracy never seen before in the field.
\end{abstract}

Trial registration: ClinicalTrials.gov NCT02231060 (registered 29 August 2014)

Keywords: Coma, Outcome prediction, Anoxic-ischemic encephalopathy, Somatosensory evoked potentials, Electroencephalogram, Neuron specific enolase, Coma recovery scale, Self-fulfilling prophecy, Observational prospective cohort study

\footnotetext{
* Correspondence: alopezr@med.Imu.de

'Department of Neurology, University of Munich, Marchioninistr. 15, Munich

D-81377, Germany

Full list of author information is available at the end of the article
} 


\section{Background}

A rapidly aging population constantly presents challenges to society in general and the health care system in particular. One such challenge is the increasing number of patients resuscitated after cardiac arrest (CA) who suffer from anoxic-ischemic encephalopathy (AIE) due to global cerebral anoxia [1]. There are approximately 80,000 such patients every year in Germany alone, which gives the issue a very high epidemiological and economic relevance [2,3]. Faced with ethical concerns and the problem of limited resources, specialists in neurocritical care use a series of tests during the first two weeks after admission to predict outcome in anoxic coma. These tests are focused on the prediction of a poor outcome. Current criteria define poor outcome as either death or unconsciousness after 1 month, or unconsciousness or severe disability after 6 months. According to these criteria, poor outcome could be accurately predicted by the following factors: "the presence of myoclonus status epilepticus on day 1 , the bilateral absence of the N20 wave of somatosensory evoked potentials (SSEPs) or a blood concentration of neuron specific enolase (NSE) above $33 \mathrm{mcg} / \mathrm{L}$ at days $1-3$, and absent pupillary and corneal reflexes or a motor response no better than extension (M1 -2) at day 3" [4, 5]. However, it can be argued that these parameters need to be revised because they are based on data that predate the widespread implementation of mild therapeutical hypothermia after cardiopulmonary resuscitation (CPR) and because data from our own laboratory suggest that they fail to predict prognosis accurately in a considerable number of patients. In a previous study, we examined over a six-year period the rehabilitation outcome of a cohort of 113 adult patients with AIE following cardiac arrest [4]. These patients had chronic disorders of consciousness at the time of admission for inpatient neurorehabilitation. We found that $25 \%$ of patients, who recovered consciousness and the ability to communicate during inpatient rehabilitation, had presented no sign of cortical SSEP responses during the initial phase at the intensive care unit (ICU). In other words, they were patients who fulfilled the current criteria for certain death or unresponsive wakefulness syndrome [5]. In fact, a regression model revealed that a malignant SSEP did not seem to be an independent predictor of rehabilitation outcome (i.e. functional or behavioural outcome) [4].

A systematic review and recent meta-analysis of the relevant literature on the state-of-the-art on early predictors ( $\leq 7$ days) of poor neurological outcome in adult patients, who received therapeutic hypothermia after cardiac arrest and survived, found that "a bilaterally absent N20 SSEP wave anytime, a nonreactive EEG after re-warming or a combination of absent ocular reflexes and $\mathrm{M} \leq 2$ after re-warming"could be used to predict a poor outcome with $0 \%$ false positive rates (100\% specificity; upper limit of $95 \% \mathrm{CI}<10 \%$ ) within a period equal to or shorter than 7 days after cardiac arrest [6]. However, the same review and meta-analysis reported that only in $11 \%$ of the studies treating teams were blinded from the results of the predictor being investigated. Since life-sustaining therapy is withdrawn from most patients with SSEP indicating bilateral loss of cortical potentials, it is difficult to know whether the resulting death of the patient is due to the natural course of the condition itself or to the fact that life-sustaining therapy was withdrawn too early [7]. This suggests that a self-fulfilling prophecy (i.e. falsely pessimistic predictions) could be biasing results and that current outcome prediction could be considerably less accurate than it is believed to be.

The present study "Hypoxia and Outcome Prediction in Early-Stage Coma" (HOPE) responds precisely to the need to improve current outcome prediction in anoxic coma by limiting the effects of the above-mentioned self-fulfilling prophecy. The primary objective of HOPE is to examine the accuracy of currently accepted parameters in the prediction of an outcome in AIE in general and the specificity of SSEP in particular.

HOPE does not use a narrow definition of outcome based entirely on outcome measures that focus on factors such as the extent of physical ability or functional independence in activities of daily life (ADL) [8]. We have observed that regaining consciousness and communication skills, despite functional dependence, is sometimes considered a favourable outcome by a previously comatose patient and his/her family [4]. Our own clinical experience is confirmed by a report on the subjective quality of life (QOL) of locked-in syndrome (LIS) patients with extreme motor impairment [9]. This study found that the experience of a good subjective QOL in a significant number of LIS patients was not dependent on the absence of extreme motor impairment and that depression was not dependent on its presence. In fact, the ability to communicate with others rather than the extent of physical ability was found to be crucial for a good subjective QOL in such patients.

\section{Methods}

\section{Study design}

HOPE is an ongoing 2.5-year multi-centre observational prospective cohort study with seven study visits and data entry points $\left(t_{0}-t_{6}\right)$ and a follow-up period of 12 months. The primary endpoints are completion of the follow-up period or patient death.

The main characteristics of HOPE are:

- Control for self-fulfilling prophecy in the outcome prediction process in the ICU 
- Long-term follow-up (12 months) including acute and neurorehabilitation phases

- Use of the Coma Recovery Scale - Revised Version, a sensitive measure of level of consciousness

- In-depth and systematic examination of the predictive value of SSEP, EEG and NSE

\section{Participants}

We expect to enrol 172 patients who remain comatose after CPR.

\section{Inclusion criteria}

- Age: $18-75$ years

- Cause of admission to ICU: Cardiac arrest

- GCS $<9$ at time of study enrolment ( 3 to 8 days after cardiac arrest). This includes GCS scores obtained during a temporary cessation of analgosedation of at least 30 minutes as long as only short-lasting drugs such as propofol or sufentanil were used

- Informed consent signed by legal guardian/next-of-kin

If a legal guardian has not yet been appointed by court, the closest relative is asked for consent. As soon as a legal guardian has been appointed, he or she is asked to renew consent.

\section{Exclusion criteria}

- Stroke (as it may interfere with SSEP testing)

- Pre-existing chronic disorder of consciousness

- Terminal malignant disease, as it increases the likelihood of not being alive for the 12-month follow-up

- Highly unlikely survival until the end of the 12-month follow-up due to conditions such as multi-organ failure based on the judgment of a critical care physician

- Existing advanced directive that demands the cessation of therapy/life support

- Withdrawal of life-support/palliative care during treatment in the ICU

- Use of barbiturates for sedation

- Inability to determine GCS $<9$ (see inclusion criteria) due to prolonged deep analgosedation

\section{Recruitment sources}

We established a study consortium consisting of five major tertiary care hospitals with cardiology ICUs and neurology departments as well as four neurorehabilitation centres in the south of the German state of Bavaria receiving patients from these ICUs.

During the acute phase, we screen CA patients admitted to cardiology ICUs. Neurological prognostic testing is assigned to the neurology department of the same hospital. We transfer patients to a neurorehabilitation centre part of HOPE once the decision for neurorehabilitation is made and the legal representatives of the patients agree. It must be noted that patients or their legal guardians are allowed to select a non-participating rehabilitation centre. When that is the case or neurorehabilitation is not advised, study visits planned for the rehabilitation phase (i.e. $t_{3}-t_{5}$ ) are not carried out. However, we conduct as planned a 12-month follow-up at the place of residence of the patient regardless of whether the patient received study visits after being discharged from the ICU or not.

\section{Measures}

In addition to collecting clinical and demographic data in accordance with Utstein-style recommendations, we assess the current level of consciousness, relevant neuropsychological functions, health-related quality of life (HRQOL) as well as information on functional outcome and activities of daily living (ADL) [10]. Table 1 details the complete assessment catalogue with its respective instruments.

The most crucial part of our study is determining the current level of consciousness. We used for that purpose the revised version of the Coma Recovery Scale (CRS-R) [11]. The CRS-R allows a precise categorisation of the current level of consciousness based on a structured and systematic clinical examination of auditory, visual, motor, oromotor, communication, and arousal functions.

\section{Data collection}

Study centres collect data prospectively following the data acquisition timeline in Table 2. Visits $t_{0}$ to $t_{2}$ take place at ICUs; visits $t_{3}$ to $t_{5}$ at the neurorehabilitation centres; and visit t6 at the location of residence of the patient 12 months after day 0.

\section{Biostatistical concept and statistical analysis}

Determining whether an outcome is truly poor requires making a distinction between functional and behavioural outcome as well as input from caregivers on what the patient would have wanted. We ask family members or significant others at the time of patient enrolment at the ICU what the patient would have considered a favourable outcome. In other words, given the possibility of a permanent unresponsive wakefulness syndrome, would the patient have considered either a functional outcome such as being able to lead an independent life or a behavioural outcome such as regaining functional communication skills the only acceptable favourable outcome.

Therefore, we use in our analysis functional and behavioural definitions of favourable outcome. In practical terms this implies the analysis of the positive predictive value of dichotomous prognostic markers (e.g. a favourable or unfavourable SSEP). 
Table 1 Assessment categories and instruments

\begin{tabular}{|c|c|}
\hline Assessment category & Instruments \\
\hline Sociodemographic factors & Age, gender, marital status, highest educational degree \\
\hline Comorbidity & Modified Cumulative IIIness Rating Scale (mCIRS) $[20,21]$. \\
\hline Severity of illness & Simplified Acute Physiology Score (SAPS II) [22, 23]. \\
\hline Prognostication tests & EEG, median-nerve somatosensory evoked potentials (SEP), NSE \\
\hline Activities of daily living (ADL) Outcome & Barthel index and the Barthel index for Early Rehabilitation (BI) [23-26]. \\
\hline - Level of consciousness & $\begin{array}{l}\text { Coma recovery scale -revised (CRS-R) [11], Glasgow Coma Scale (GCS) [27], Full outline of } \\
\text { unresponsiveness (FOUR) [28] }\end{array}$ \\
\hline - Health status & $\begin{array}{l}\text { Neurological post-acute Core Set of the International Classification of Functioning, } \\
\text { Disability and Health (ICF) [29] }\end{array}$ \\
\hline - Cognitive functioning & $\begin{array}{l}\text { Since the most commonly observed cognitive impairments in patients with an anoxic-ischemic } \\
\text { brain injury are disturbances of attention/processing speed, memory, and executive function } \\
\text { we use the subtests of the Cologne Neuropsychological Screening for Stroke Patients (German: } \\
\text { Koelner Neuropsychologisches Screening für Schlaganfall-Patienten) for these functions [30, 31]. }\end{array}$ \\
\hline - Psychosocial Outcome & German version of the Hospital Anxiety and Depression Scale (HADS-D) [32-34]. \\
\hline - Quality of life & Short Form 36 (SF-36) Questionnaire (for patients as well as for closest care-providing relative) [35] \\
\hline $\begin{array}{l}\text { - Life Satisfaction (LS) and satisfaction } \\
\text { with decision making at the ICU }\end{array}$ & $\begin{array}{l}\text { The LS of the patient and the closest care-providing person is measured by the "Question } \\
\text { on Life Satisfaction (German: Fragen zur Lebenszufriedenheit-Module; FLZ-M) [36], and } \\
\text { satisfaction with decision making at the ICU is measured with questions such as } \\
\text { "in retrospective, I would have decided exactly the same on the ICU" using a 5-point scale. }\end{array}$ \\
\hline - Overall outcome & The modified Rankin scale (mRS) $[37,38]$, and the Cerebral Performance Category $[39,40]$ \\
\hline
\end{tabular}

In the case of a definition of favourable outcome predicated upon the capacity to lead an independent daily life (i.e. functional outcome), we use both a Barthel-Index score and a Modified Rankin Scale (mRS) score to quantify and categorise a favourable functional outcome. However, in the case of a favourable outcome defined in terms of the extent of the recovery of consciousness (i.e. behavioural outcome), we analyse CRS-R scores to quantify it. It must be noted that the recovery of full consciousness as measured by the CRS-R implies the regaining of functional communication skills.

We also analyse the effect of covariates adjusted for age and sex such as sociodemographic variables, prognostic variables and treatment factors (e.g. use of therapeutic hypothermia) on functional (i.e. Barthel-Index scores) and behavioural (i.e. CRS-R scores) outcomes with mixed effects regression models (i.e. fixed and random effects). This method of analysing longitudinal data has been reported to be highly effective to examine

Table 2 Data acquisition timeline and type of data to be acquired during study visits/time points

\begin{tabular}{|c|c|c|c|c|c|c|c|}
\hline \multicolumn{8}{|l|}{ Timeline } \\
\hline & to: & t1: & t2: & t3: & t4: & t5: & t6: \\
\hline & Screening & Days 3-8 & Days 8-14 & Admission Rehab & Rehab week 4 & Discharge Rehab & Follow-Up 1 year \\
\hline Location of data collection & ICU & ICU & ICU & Rehab & Rehab & Rehab & Home \\
\hline \multicolumn{8}{|l|}{ Assessment categories } \\
\hline - Inclusion/Exclusion criteria & $\checkmark$ & & & & & & \\
\hline - Sociodemographic factors & & $\checkmark$ & & & & & \\
\hline - Comorbidity & & $\checkmark$ & & & & & \\
\hline - Severity of illness & $\checkmark$ & & & & & & \\
\hline - Prognostication tests & & $\checkmark$ & $\checkmark$ & $\checkmark$ & & $\checkmark$ & \\
\hline - Level of consciousness & & $\checkmark$ & $\checkmark$ & $\checkmark$ & $\checkmark$ & $\checkmark$ & $\checkmark$ \\
\hline - Activities daily living & & & $\checkmark$ & $\checkmark$ & $\checkmark$ & $\checkmark$ & $\checkmark$ \\
\hline - Further patient outcome & & & & & & & $\checkmark$ \\
\hline
\end{tabular}


change trajectories with several unequally spaced waves of data $[12,13]$.

\section{Power analysis and sample size}

Clinicians typically consider that the likelihood of a poor outcome is high when they observe the following factors (i.e. negative predictors) during ICU treatment with a specificity $>90 \%$ and a confidence interval of $95 \%$ : (i) bilateral loss of cortical N20 median nerve SEP potentials, (ii) loss of at least one brainstem reflex, (iii) non-reactive EEG, (iv) or a NSE above $75 \mathrm{mcg} / \mathrm{L}$ during ICU treatment $[1,3,6,14,15]$. Based on this fact, our null hypothesis is that there is a probability of $<10 \%$ that a favourable outcome will be observed despite the presence of any of the predictors of a poor/ negative outcome. We should reiterate that the term "favourable outcome" in this particular context refers to the outcome most likely preferred by the patient in the opinion of his or her closest relative or significant other.

We test the null hypothesis against a one-sided alternative using a Simon's two-stage design to determine whether it is warranted to recruit the full number of patients suggested by the result of the power analysis we performed [16]. In the first stage as soon as we manage to recruit $42 \%$ (i.e. the first 72 patients) of the total number of patients needed (i.e. 172) we will analyse the data of this subset to determine whether the null hypothesis can be rejected. In practical terms this means that if $<10 \%$ of patients in this early subset present a positive outcome despite negative predictors, the study must be aborted because the null hypothesis could not be rejected. However, if we observe that $>10 \%$ do present a favourable outcome despite negative predictors, we would proceed to the second stage of the study, namely, the recruitment of the full number of patients necessary to achieve sufficient statistical power (i.e. 172). In this second stage we adopt a design that yields a Type I error rate of 0.05 and $80 \%$ power if the true response rate is $25 \%$.

The null hypothesis will be rejected if 28 or more favourable outcomes are observed in these 172 patients. That is, if $25 \%$ or more patients do present a positive outcome despite a negative predictor. This calculation is based on the assumption that $25 \%$ of enrolled patients have at least one negative predictor. This is a conservative assumption if we consider previous studies from our own research group $[4,11]$.

\section{Control for sampling bias and self-fulfilling prophecy Sampling bias}

In order to avoid sampling bias, we screen consecutively CA patients admitted to one of the participating intensive care units (ICU) based on the inclusion and exclusion criteria of the study. Screening logs are filled out for all CA patients admitted to these ICUs and include specific reasons for excluding a given patient.

\section{Bias due to self-fulfilling prophecy}

As explained above, life-sustaining therapy is withdrawn from most patients with bilateral loss of cortical SSEP responses. It is consequently difficult to know whether the resulting death of the patient is due to the natural course of the condition or to the fact that life-sustaining therapy was withdrawn too early [7]. Therefore, most cardiac arrest outcome studies can be considered heavily biased due to a self-fulfilling prophecy.

To control for this bias we implement the following measures:

a) All ICU team members involved in counselling of relatives and strategic decision-making are made aware of the existing conflicting data on prognostic test accuracy.

b) Study information material and informed consent forms contain information on the likelihood of recovery of consciousness and functional independence depending on the presence of negative predictors.

c) Study personnel from the study coordination centre are available to ICUs in the study for additional counselling and realistic expectation management of relatives and family decision makers.

d) As part of the battery of case report forms (CRF), we provide a standardised prognostication form for ICU physicians containing a multimodal procedure based on the recommendations in the literature [17]. Its purpose is to lend perspective to prognostic test results such as brain stem reflexes, EEG, SSEP, and NSE so that they can convey as accurately as possible to patients' surrogates the degree of prognostic uncertainty and the likelihood of a given outcome. Physicians are prompted to select one of the semi-quantitative conclusions shown in Fig. 1.

e) Patients are excluded from the study if life-sustaining therapy is withdrawn or if pre-existing advance directives call for palliative care.

It must be noted that ICU clinical decision makers are asked to follow their best ethical judgment at all times and are therefore free to follow treatment strategies that do not adhere to the study protocol.

\section{Bias due to quality control issues}

Study staff receives assessment training and retraining regularly. The study coordination centre supervises assessments and provides a manual and a data dictionary 


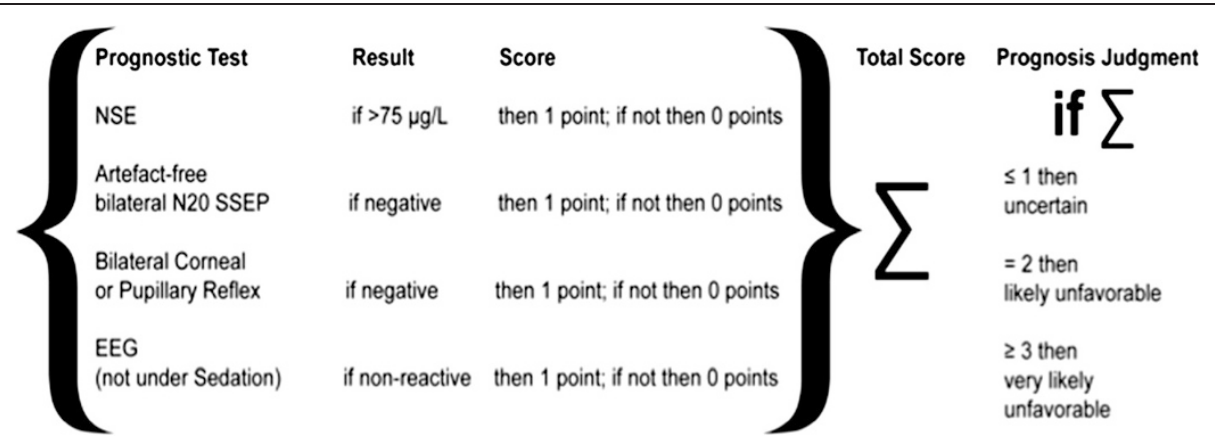

Fig. 1 Proposed multimodal prognostic approach to avoid falsely pessimistic outcome predictions

to all study centres along with CRFs. Study centres in turn check data before submission for completeness, plausibility, accuracy, consistency and outliers.

\section{Ethical considerations}

As HOPE is a non-interventional trial its examinations are part of the clinical standard operating procedures at participating centres and comply with the guidelines of the German Neurological Society (Deutsche Gesellschaft für Neurologie; DGN) for AIE. HOPE was approved by the ethics committee of the University of Munich and is funded by the Else Kroene-Fresenius Foundation, a nonprofit-organisation that supports medical and humanitarian projects through a peer-review process.

HOPE endeavours to ensure that patients do not experience death due to withdrawal of care. However, medical strategy regarding continued versus palliative care or withdrawal of care is solely dependent on the decision of patient surrogates and senior ICU physicians and not on study protocol.

\section{Study limitations}

Since HOPE is an observational study, there is no intervention and medical decision-making is at the sole discretion of attending physicians. The latter fact has a direct impact on the implementation of the recommended course of action to control for the effect of a self-fulfilling prophecy during the outcome prediction process at the ICU. Physicians are free to accept or ignore project directives in that regard. However, ignoring project recommendations to avoid a self-fulfilling prophecy renders the data of the patient in question unusable for the purposes of the project. As this may slow down data collection, we seek to heighten the engagement of physicians at project sites by offering training workshops, a frequent newsletter, briefings by the leading investigator and the project manager as well as updates on the condition of patients even after they leave the ICU. This is undertaken while emphasizing at all times that the objectives of the project must not influence medical strategy.
Another possible limitation of HOPE is that it may be affected by the "ICU variable", namely regional differences in healthcare provision (e.g. infrastructure and organisation specific to Germany) [18]. However, since the outcome prediction parameters being examined by the study are part of routine basic examinations at ICUs around the world, it is safe to assume that the ICU variable is not likely to limit the generalisation of results.

\section{Discussion}

To our best knowledge, HOPE is the first observational multicentre prospective cohort study on coma prognosis to control for the effect of a possible self-fulfilling prophecy at the ICU and to cover the acute and neurorehabiltation phases with a long-term follow-up longer than the usual three or six months. Follow-up accuracy is ensured by the fact that, unlike most previous studies, we employ the CRS-R, a behavioural scale more sensitive than the Glasgow Coma Scale. We strongly believe that we will be able to make a significant contribution to the revision and improvement of current outcome prediction methods in anoxic-ischemic encephalopathy (AIE) patients. As a result, neurocritical care specialists worldwide will have considerably more accurate methods for prognosticating the outcome of AIE following cardiac arrest. This will facilitate the provision of treatment tailored to individual patients and the attainment of an optimal QOL. It will also inform the decision to withdraw treatment with a level of accuracy never seen before in the field.

In a Time Magazine article, which offered the general public a rare accurate look into cutting-edge research on disorders of consciousness, we find a poignant question. After referring to patient cases that illustrate the difficulty of predicting an outcome, the author asks candidly: “... patients labelled vegetative typically stay that way-but sometimes they don't. So where's the line between resignation and hope?" [19]. We believe that HOPE will make a significant contribution to bringing us considerably closer to finding the answer to this important question. 


\section{Competing interests}

The authors declare that they have no competing interests.

\section{Authors' contributions}

$A L-R$ and $A B$ contributed substantially to the study design and methods, drafted the manuscript and revised it critically. All authors read and approved the final manuscript.

\section{Acknowledgment}

The authors wish to thank the Else Kröne-Fresenius Stiftung for its generous funding of Project HOPE. We are particularly grateful to Ulrich Mansmann for contributing to the planning of the statistical analysis and power calculation as well as to Jonathan Shock for contributing to the revision of a part of the manuscript. In addition to the authors of the present paper, the Project HOPE Investigator Group consists of: Andreas Straube, Ulrich Mansmann, Stefanie Förderreuther, Philipp Lange, Daniel Braun, Philipp Baumann, Jan Rémi, Matthias Angstwurm, Suzette Heck, Mareike Wengerofsky and Stefan Czermak in the University of Munich, Jonathan Shock in the University of Cape Town, Thomas Pfefferkorn, Karlheinz Seidl, Ana-Lioara Arva, and Mohammad Hujeirat in Klinikum Ingolstadt, Hanns Lohner and Christine Kummer in RoMed Klinikum Rosenheim, Jürgen Herzog in Schön Klinik München Schwabing, Friedemann Müller and Barbara Schäpers and Maria Schlutt in Schön Klinik Bad Aibling, Marion Arndt, and Barbara Schorr in Therapiezentrum Burgau, Rüdiger Ilg in Asklepios Stadtklinik Bad Tölz, Markus Naumann, Konrad Scheglmann, Andreas Blüthgen and Wolfgang von Scheidt in Klinikum Augsburg.

\section{Author details}

${ }^{1}$ Department of Neurology, University of Munich, Marchioninistr. 15, Munich D-81377, Germany. ${ }^{2}$ Therapiezentrum Burgau, Burgau, Germany.

\section{Received: 1 April 2015 Accepted: 5 May 2015 \\ Published online: 15 May 2015}

\section{References}

1. Diener H-C. Leitlinien für Diagnostik und Therapie in der Neurologie: Herausgegeben von der Kommission "Leitlinien" der DGN. Auflage: 5. vollständig überarbeitete Auflage. Stuttgart: Thieme; 2012.

2. Graf J, Mühlhoff C, Doig GS, Reinartz S, Bode K, Dujardin R, et al. Health care costs, long-term survival, and quality of life following intensive care unit admission after cardiac arrest. Crit Care Lond Engl. 2008;12:R92.

3. Wijdicks EFM, Hijdra A, Young GB, Bassetti CL, Wiebe S, Quality Standards Subcommittee of the American Academy of Neurology. Practice parameter: prediction of outcome in comatose survivors after cardiopulmonary resuscitation (an evidence-based review): report of the Quality Standards Subcommittee of the American Academy of Neurology. Neurology. 2006;67:203-10.

4. Howell K, Grill E, Klein A-M, Straube A, Bender A. Rehabilitation outcome of anoxic-ischaemic encephalopathy survivors with prolonged disorders of consciousness. Resuscitation. 2013;84:1409-15.

5. Laureys S, Celesia GG, Cohadon F, Lavrijsen J, León-Carrión J, Sannita WG, et al. Unresponsive wakefulness syndrome: a new name for the vegetative state or apallic syndrome. BMC Med. 2010;8:68.

6. Sandroni C, Cavallaro F, Callaway CW, D'Arrigo S, Sanna T, Kuiper MA, et al. Predictors of poor neurological outcome in adult comatose survivors of cardiac arrest: a systematic review and meta-analysis. Part 2: Patients treated with therapeutic hypothermia. Resuscitation. 2013;84:1324-38.

7. Geocadin RG, Buitrago MM, Torbey MT, Chandra-Strobos N, Williams MA, Kaplan PW. Neurologic prognosis and withdrawal of life support after resuscitation from cardiac arrest. Neurology. 2006;67:105-8.

8. Rittenberger JC, Raina K, Holm MB, Kim YJ, Callaway CW. Association between Cerebral Performance Category, Modified Rankin Scale, and Discharge Disposition after Cardiac Arrest. Resuscitation. 2011;82:1036-40.

9. Lulé D, Zickler C, Häcker S, Bruno MA, Demertzi A, Pellas F, et al. Life can be worth living in locked-in syndrome. Prog Brain Res. 2009;177:339-51.

10. Jacobs I, Nadkarni V, Bahr J, Berg RA, Billi JE, Bossaert L, et al. Cardiac arrest and cardiopulmonary resuscitation outcome reports: update and simplification of the Utstein templates for resuscitation registries: a statement for healthcare professionals from a task force of the International Liaison Committee on Resuscitation (American Heart Association, European Resuscitation Council, Australian Resuscitation Council, New Zealand Resuscitation Council, Heart and Stroke Foundation of Canada,
InterAmerican Heart Foundation, Resuscitation Councils of Southern Africa). Circulation. 2004;110:3385-97.

11. Giacino JT, Kalmar K, Whyte J. The JFK Coma Recovery Scale-Revised: measurement characteristics and diagnostic utility. Arch Phys Med Rehabil. 2004;85:2020-9.

12. Liu LC. A model for incomplete longitudinal multivariate ordinal data. Stat Med. 2008;27:6299-309.

13. Liu LC, Hedeker D. A mixed-effects regression model for longitudinal multivariate ordinal data. Biometrics. 2006;62:261-8.

14. Bouwes A, Binnekade JM, Kuiper MA, Bosch FH, Zandstra DF, Toornvliet AC et al. Prognosis of coma after therapeutic hypothermia: a prospective cohort study. Ann Neurol. 2012;71:206-12.

15. Rossetti AO, Oddo M, Logroscino G, Kaplan PW. Prognostication after cardiac arrest and hypothermia: a prospective study. Ann Neurol. 2010;67:301-7.

16. Simon R. Optimal two-stage designs for phase II clinical trials. Control Clin Trials. 1989;10:1-10.

17. Pistoia F, Sacco S, Stewart JE, Carolei A. Postanoxic vegetative state: avoiding the self-fulfilling prophecy. Neurohospitalist. 2015;5:7.

18. Thijs LGG. Sibbald WJ, Bion JF. In: Sibbald WJ, Bion JF, editors. Evaluating Critical Care. Berlin, Heidelberg: Springer; 2002. p. 292-308 [Update in Intensive Care Medicine, vol. 35].

19. Harrell E. A flicker of consciousness. Time. 2011;178:42-7.

20. Linn BS, Linn MW, Gurel L. Cumulative illness rating scale. J Am Geriatr Soc. 1968; 16:622-6.

21. Salvi F, Miller MD, Grilli A, Giorgi R, Towers AL, Morichi V, et al. A Manual of Guidelines to Score the Modified Cumulative Illness Rating Scale and Its Validation in Acute Hospitalized Elderly Patients. J Am Geriatr Soc. 2008;56:1926-31.

22. Capuzzo M, Moreno RP, Le Gall J-R. Outcome prediction in critical care: the Simplified Acute Physiology Score models. Curr Opin Crit Care. 2008;14:485-90.

23. Le Gall JR, Lemeshow S, Saulnier F. A new Simplified Acute Physiology Score (SAPS II) based on a European/North American multicenter study. JAMA. 1993;270:2957-63.

24. Mahoney Fl, Barthel DW. Functional Evaluation: The Barthel Index. Md State Med J. 1965;14:61-5.

25. Granger CV, Dewis LS, Peters NC, Sherwood CC, Barrett JE. Stroke rehabilitation: analysis of repeated Barthel index measures. Arch Phys Med Rehabil. 1979:60:14-7.

26. Arbeitsgemeinschaft Neurologische Frührehabilitation. The Frühreha-Index (FRI): A Manual for its Operationalization. Rehabil. 2010;49:22-9.

27. Teasdale G, Jennett B. Assessment of coma and impaired consciousness. A practical scale. Lancet. 1974:2:81-4.

28. Chen B, Grothe C, Schaller K. Validation of a new neurological score (FOUR Score) in the assessment of neurosurgical patients with severely impaired consciousness. Acta Neurochir (Wien). 2013;155:2133-9. discussion 2139.

29. Grill E, Strobl R, Müller M, Quittan M, Kostanjsek N, Stucki G. ICF Core Sets for early post-acute rehabilitation facilities. J Rehabil Med. 2011;43:131-8.

30. Anderson CA, Arciniegas DB. Cognitive sequelae of hypoxic-ischemic brain injury: a review. NeuroRehabilitation. 2010;26:47-63.

31. Kaesberg S, Fink G, Kalbe E. Neuropsychologische Frühdiagnostik nach einem Schlaganfall - ein Überblick im deutschsprachigen Raum verfügbarer Instrumente und Vorstellung eines neuen Screening-Verfahrens. Fortschritte Neurol . Psychiatr. 2013;81:482-92.

32. Zigmond AS, Snaith RP. The Hospital Anxiety and Depression Scale. Acta Psychiatr Scand. 1983;67:361-70.

33. Herrmann C. International experiences with the Hospital Anxiety and Depression Scale-A review of validation data and clinical results. J Psychosom Res. 1997:42:17-41.

34. Hinz A, Brähler E. Normative values for the Hospital Anxiety and Depression Scale (HADS) in the general German population. J Psychosom Res. 2011;71:74-8.

35. McHorney CA, Ware JE, Lu JF, Sherbourne CD. The MOS 36-item Short-Form Health Survey (SF-36): III. Tests of data quality, scaling assumptions, and reliability across diverse patient groups. Med Care. 1994;32:40-66.

36. Henrich G, Herschbach P. Questions on Life Satisfaction (FLZ-M) - A Short Questionnaire for Assessing Subjective Quality of Life. Eur J Psychol Assess. 2000;16:150-9.

37. Rankin J. Cerebral vascular accidents in patients over the age of 60. II. Prognosis. Scott Med J. 1957;2:200-15.

38. Wilson JTL, Hareendran A, Hendry A, Potter J, Bone I, Muir KW. Reliability of the modified Rankin Scale across multiple raters: benefits of a structured interview. Stroke J Cereb Circ. 2005;36:777-81. 
39. Jennett B, Bond $\mathrm{M}$. Assessment of outcome after severe brain damage. Lancet. 1975;1:480-4.

40. Ajam K, Gold LS, Beck SS, Damon S, Phelps R, Rea TD. Reliability of the Cerebral Performance Category to classify neurological status among survivors of ventricular fibrillation arrest: a cohort study. Scand J Trauma Resusc Emerg Med. 2011;19:38.

Submit your next manuscript to BioMed Central and take full advantage of:

- Convenient online submission

- Thorough peer review

- No space constraints or color figure charges

- Immediate publication on acceptance

- Inclusion in PubMed, CAS, Scopus and Google Scholar

- Research which is freely available for redistribution 